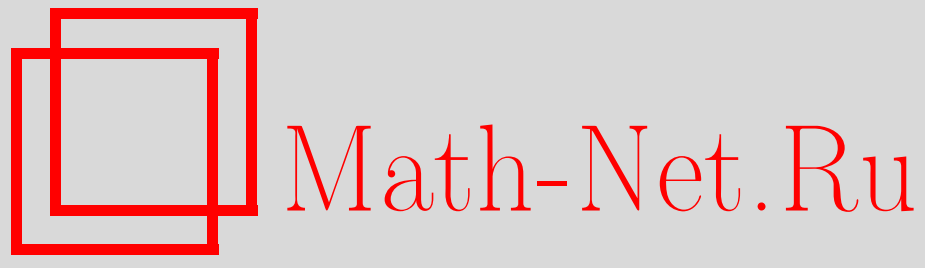

Л. Г. Рыбников, О коммутативности слабо коммутативных римановых однородных пространств, Функи. анализ и его прил., 2003, том 37, выпуск 2, 41-51

DOI: https://doi.org/10.4213/faa147

Использование Общероссийского математического портала Math-Net.Ru подразумевает, что вы прочитали и согласны с пользовательским соглашением

http://www.mathnet.ru/rus/agreement

Параметры загрузки:

IP : 54.210 .77 .194

26 апреля 2023 г., 03:28:03

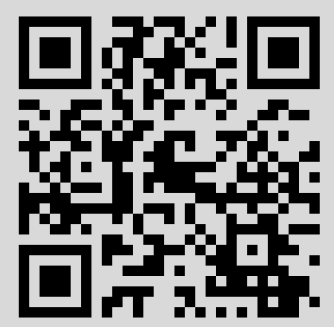


Функциональный анализ и его приложения

2003, т. 37, вып. 2, с. 41-51

УДК 514.75

\title{
О коммутативности слабо коммутативных римановых однородных пространств*
}

\author{
(c) 2003. Л. Г. РыБников
}

\section{§1. Введение}

Пусть $G$ - вещественная группа Ли, $\mathfrak{g}=\operatorname{Lie} G, H-$ компактная подгруппа в $G$ и $\mathfrak{h}=$ Lie $H$. Рассмотрим риманово однородное пространство $X=G / H$. Пусть $D(X)$ - алгебра дифференциальных операторов на $X$.

На алгебре $D(X)$ имеется фильтрация, $D(X)=\bigcup_{n=0}^{\infty} D(X)^{(n)}$, по порядку дифференциального оператора. Соответствующая градуированная алгебра $\operatorname{gr} D(X)$ изоморфна алгебре $P(X)$ функций на кокасательном расслоении $T^{*} X$ многообразия $X$, полиномиальных на слоях, причем изоморфизм осуществляется взятием символа дифференциального оператора.

На алгебре $P(X)$ естественно возникает скобка Пуассона: при $r \in D(X)^{(n)}$, $s \in D(X)^{(m)}$ имеем $\left\{\mathrm{gr}_{n} r, \mathrm{gr}_{m} s\right\}=\operatorname{gr}_{n+m-1}[r, s]$. Она совпадает со скобкой Пуассона, определяемой стандартной симплектической структурой на $T^{*} X$. Основными объектами дальнейших рассмотрений будут алгебры $D(X)^{G}$ и $P(X)^{G}-$ подалгебры $G$-инвариантов в $D(X)$ и $P(X)$ соответственно. Очевидно, что пуассонова алгебра, соответствующая алгебре $D(X)^{G}$, вложена в алгебру $P(X)^{G}$. Дадим более явное описание алгебры $D(X)^{G}$ в терминах универсальной обертывающей алгебры $U(\mathfrak{g})$.

Каждому элементу $\xi$ алгебры Ли $\mathfrak{g}=T_{e} G$ поставим в соответствие левоинвариантный дифференциальный оператор первого порядка на группе $G$, являющийся дифференцированием по направлению левоинвариантного векторного поля, равного $\xi$ в единице группы $G$. Это отображение продолжается до изоморфизма алгебры $U(\mathfrak{g})$ на алгебру левоинвариантных дифференциальных операторов на $G$. При этом порядок дифференциального оператора, соответствующего элементу $u \in U(\mathfrak{g})$, равен степени выражения элемента $u$ через элементы алгебры Ли $\mathfrak{g}$, а взятие символа левоинвариантного дифференциального оператора в точке $e$ есть изоморфизм $\operatorname{gr} U(\mathfrak{g}) \rightarrow S(\mathfrak{g})$, описываемый теоремой ПуанкареБиркгофа-Витта.

Пусть $U(\mathfrak{g})^{H}$ - подалгебра алгебры $U(\mathfrak{g})$, состоящая из элементов, инвариантных относительно присоединенного действия подгруппы $H$. Аналогично определим подалгебру $S(\mathfrak{g})^{H}$ в $S(\mathfrak{g})$. Алгебры $U(\mathfrak{g})$ и $S(\mathfrak{g})$ являются изоморфными $H$-модулями (более того, изоморфными $G$-модулями), а следовательно, вложение $\operatorname{gr} U(\mathfrak{g})^{H} \rightarrow S(\mathfrak{g})^{H}$ является изоморфизмом. Всякий оператор $D \in U(\mathfrak{g})^{H}$ перестановочен с правыми сдвигами на элементы подгруппы $H$ и, следовательно, переводит в себя пространство функций на $G$, правоинвариантных относительно $H$. Тем самым он определяет некоторый инвариантный дифференци-

* Работа выполнена при поддержке фонда CRDF, грант RMI-2088. 
альный оператор $D_{H}$ на многообразии $X=G / H$. Отметим, что символ оператора $D_{H}$ есть образ символа оператора $D$ при каноническом гомоморфизме $P\left(T^{*} G\right)^{G} \rightarrow P\left(T^{*} X\right)^{G}$. В частности, символ оператора $D_{H}$ в точке $o=e H \in X$ есть образ символа оператора $D$ в точке $e \in G$ при каноническом гомоморфизме $\mathbb{R}\left[T_{e}^{*} G\right]=S(\mathfrak{g}) \rightarrow S(\mathfrak{g} / \mathfrak{h})=\mathbb{R}\left[T_{o}^{*} X\right]$.

Все дифференциальные операторы из левого идеала $U(\mathfrak{g}) \mathfrak{h}$ алгебры $U(\mathfrak{g})$ аннулируют функции, правоинвариантные относительно $H$. Так как элементы подалгебры $U(\mathfrak{g})^{H}$ перестановочны с элементами из $\mathfrak{h}$, то $(U(\mathfrak{g}) \mathfrak{h})^{H}-$ двусторонний идеал алгебры $U(\mathfrak{g})^{H}$. Таким образом, определен гомоморфизм ассоциативных алгебр

$$
\mu: U(\mathfrak{g})^{H} /(U(\mathfrak{g}) \mathfrak{h})^{H} \rightarrow D(X)^{G} .
$$

Так как группа $H$ компактна, то $U(\mathfrak{g}) \mathfrak{h}$ и $S(\mathfrak{g}) \mathfrak{h}$ являются изоморфными $H$-модулями. Следовательно, $\operatorname{gr}(U(\mathfrak{g}) \mathfrak{h})^{H}=(S(\mathfrak{g}) \mathfrak{h})^{H}$. Отсюда, в частности, вытекает, что $(S(\mathfrak{g}) \mathfrak{h})^{H}-$ пуассонов идеал в алгебре $S(\mathfrak{g})^{H}$. Переходя от ассоциативных алгебр к ассоциированным пуассоновым, получаем гомоморфизм пуассоновых алгебр

$$
\operatorname{gr} \mu: S(\mathfrak{g})^{H} /(S(\mathfrak{g}) \mathfrak{h})^{H} \rightarrow \operatorname{gr} D(X)^{G} \subset P(X)^{G} .
$$

Известно [1], что

1) гомоморфизм $\mu$ является изоморфизмом ассоциативных алгебр,

$$
U(\mathfrak{g})^{H} /(U(\mathfrak{g}) \mathfrak{h})^{H} \simeq D(X)^{G} ;
$$

2) гомоморфизм gr $\mu$ является изоморфизмом пуассоновых алгебр,

$$
S(\mathfrak{g})^{H} /(S(\mathfrak{g}) \mathfrak{h})^{H} \simeq P(X)^{G} .
$$

Отсюда следует, что пуассонова алгебра, соответствующая алгебре $D(X)^{G}$, совпадает с алгеброй $P(X)^{G}$.

Изоморфизм пуассоновых алгебр

$$
(\operatorname{gr} \mu)^{-1}: P(X)^{G} \rightarrow S(\mathfrak{g})^{H} /(S(\mathfrak{g}) \mathfrak{h})^{H}=\mathbb{R}\left[T_{o}^{*} X\right]^{H}
$$

имеет очевидную геометрическую интерпретацию: это гомоморфизм ограничения на слой $T_{o}^{*} X=(\mathfrak{g} / \mathfrak{h})^{*}$ кокасательного расслоения в точке $o=e H$.

Однородное пространство $X$ называется коммутативныц, если алгебра $D(X)^{G}$ коммутативна, и слабо коммутативным, если алгебра $P(X)^{G}$ коммутативна относительно скобки Пуассона. Так как $\operatorname{gr} D(X)^{G}=P(X)^{G}$, то из коммутативности риманова однородного пространства следует его слабая коммутативность.

Вопрос об истинности обратного утверждения, т.е. о коммутативности слабо коммутативных однородных пространств, тесно связан с гипотезой М. Дюфло [4], состоящей в следующем. Пусть $G-$ связная группа Ли и $H-$ связная подгруппа в $G$. Для любого характера $\nu$ группы $H$ обозначим через $\mathfrak{h}^{\nu}$ подпространство в $U(\mathfrak{g})$ или $S(\mathfrak{g})$, являющееся линейной оболочкой элементов $y+\nu(y), y \in \mathfrak{h}$. Определим характер $\delta$ группы $H$ по формуле $\delta(h)=\left(\operatorname{det}_{\mathfrak{g} / \mathfrak{h}} \operatorname{Ad} h\right)^{1 / 2}$.

Пусть $\lambda-$ произвольный характер группы $H$. Рассмотрим следующие фильтрованные коммутативные алгебры:

1) $Z\left(S(\mathfrak{g}) / S(\mathfrak{g}) \mathfrak{h}^{\lambda}\right)^{H}$ - центр пуассоновой алгебры $\left(S(\mathfrak{g}) / S(\mathfrak{g}) \mathfrak{h}^{\lambda}\right)^{H}$;

2) $Z\left(U(\mathfrak{g}) / U(\mathfrak{g}) \mathfrak{h}^{\lambda-\delta}\right)^{H}$ - центр ассоциативной алгебры $\left(U(\mathfrak{g}) / U(\mathfrak{g}) \mathfrak{h}^{\lambda-\delta}\right)^{H}$. 
Ассоциированные градуированные алгебры каноническим образом вкладываются в градуированную алгебру $(S(\mathfrak{g}) / S(\mathfrak{g}) \mathfrak{h})^{H}$.

ГипотезА. Существует отображение

$$
\beta: Z\left(S(\mathfrak{g}) / S(\mathfrak{g}) \mathfrak{h}^{\lambda}\right)^{H} \rightarrow Z\left(U(\mathfrak{g}) / U(\mathfrak{g}) \mathfrak{h}^{\lambda-\delta}\right)^{H},
$$

осуществляющее изоморфизм фильтрованных алгебр, такое, ито следующая диаграмма коммутативна:

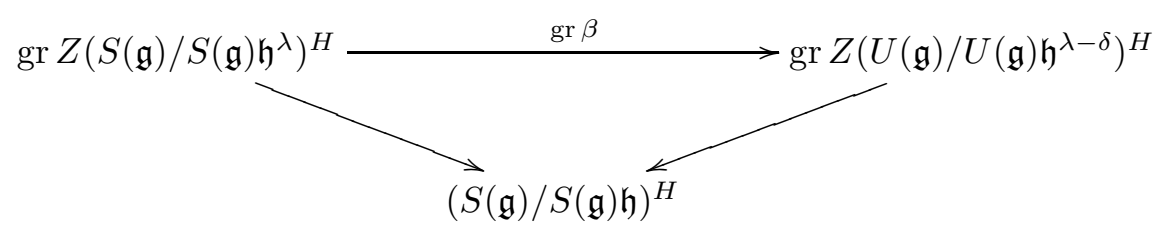

При $H=\{e\}$ эта гипотеза превращается в известную теорему Дюфло (см., например, [2]) об изоморфизме центра универсальной обертывающей алгебры $U(\mathfrak{g})$ и центра пуассоновой алгебры $S(\mathfrak{g})$. В случае редуктивных групп $G$ и $H$ при $\lambda=0$ эта гипотеза следует из результатов Кнопа [6]. В общем случае вопрос остается открытым.

Если группа $H$ компактна, то характер $\delta$ тривиален. Таким образом, в этом случае гипотеза Дюфло означает изоморфизм центра ассоциативной алгебры $(U(\mathfrak{g}) / U(\mathfrak{g}) \mathfrak{h})^{H}=D(X)^{G}$ и центра пуассоновой алгебры $(S(\mathfrak{g}) / S(\mathfrak{g}) \mathfrak{h})^{H}=P(X)^{G}$. В частности, если пуассонова алгебра $P(X)^{G}$ совпадает со своим центром, то ассоциативная алгебра $D(X)^{G}$ тоже совпадает со своим центром. Таким образом, коммутативность слабо коммутативных римановых однородных пространств следует из гипотезы Дюфло для случая $\lambda=0$ и компактной группы $H$. Однако даже в этом случае при произвольной группе $G$ вопрос об истинности гипотезы остается открытым.

Известно, что утверждение о коммутативности слабо коммутативных римановых однородных пространств верно в двух противоположных случаях: когда группа $G$ редуктивна $[3,5]$ (в этом случае пространство $X$ является сферическим) и когда $G=H \curlywedge N$, где $N-$ нильпотентная группа Ли [1]. В настоящей работе это утверждение будет доказано в общем случае. В том числе это даст новое доказательство в случае редуктивной группы $G$.

Основная идея доказательства состоит в явном построении «достаточно большой» коммутативной подалгебры в $D(X)^{G}$. В случае редуктивной группы $G$ эта подалгебра совпадает с образом центра универсальной обертывающей алгебры при отображении $\mu$.

Я благодарю Э. Б. Винберга за постановку задачи и постоянное внимание к работе. Я благодарен также рецензенту за ценные замечания.

\section{§2. Некоторые обозначения}

$Z A$ - центр ассоциативной алгебры $A$.

$\operatorname{Quot}(B)$ - поле частных целостной коммутативной алгебры $B$.

$F[X]$ - алгебра регулярных (полиномиальных) функций на алгебраическом многообразии $X$ над полем $F$.

$F(X)$ - алгебра рациональных функций на алгебраическом многообразии $X$ над полем $F$. 
$T_{x}(X)$ - касательное пространство многообразия $X$ в точке $x$.

$\mathfrak{h}^{\circ}\left(\simeq(\mathfrak{g} / \mathfrak{h})^{*}\right)-$ аннулятор подпространства $\mathfrak{h} \subset \mathfrak{g}$ в $\mathfrak{g}^{*}$.

$L<N-$ полупрямое произведение групп $H$ и $N$, в котором подгруппа $N$ нормальна.

$\mathfrak{l}+\mathfrak{n}-$ полупрямая сумма алгебр Ли $\mathfrak{l}$ и $\mathfrak{n}$, в которой $\mathfrak{n}-$ идеал.

Группы Ли обозначаются прописными латинскими буквами, а их касательные алгебры Ли - соответствующими строчными готическими буквами.

\section{§3. Доказательства вспомогательных утверждений}

ЛЕмма 1. Пусть $\mathscr{A}=\bigcup_{n=0}^{\infty} \mathscr{A}_{n}-$ фильтрованная ассоизиативная алгебра u $A=\operatorname{gr} \mathscr{A}$. Пусть $\mathscr{B} \subset \mathscr{A}-$ некоторая коммутативная подалгебра, а $B=$ gr $\mathscr{B}$, причем

1) А - целостная коммутативная алгебра;

2) $A \supseteq B-$ алгебрачческое расширение.

Тогда алгебра $\mathscr{A}$ коммутативна.

ДокАЗАтельство. Рассмотрим последовательность операций $\{\cdot, \cdot\}_{k}$ на алгебре $A$, задаваемую следующим образом:

1) при $r \in \mathscr{A}_{n}, s \in \mathscr{A}_{m}$ имеем $\left\{\operatorname{gr}_{n} r, \operatorname{gr}_{m} s\right\}_{1}=\operatorname{gr}_{n+m-1}[r, s]$;

2) если операция $\{\cdot, \cdot\}_{k-1}$ нулевая, то при $r \in \mathscr{A}_{n}, s \in \mathscr{A}_{m}$ имеем $\left\{\operatorname{gr}_{n} r\right.$, $\left.\operatorname{gr}_{m} s\right\}_{k}=\operatorname{gr}_{n+m-k}[r, s]$.

Предположим, что алгебра $\mathscr{A}$ не коммутативна. Тогда эта последовательность оборвется на каком-то $l$-м шаге. Очевидно, что $\{\cdot, \cdot\}_{l}$ является нетривиальным бидифференцированием алгебры $A$, причем $\{B, B\}_{l}=0$.

Любой элемент $s \in B$ задает дифференцирование алгебры $A$, переводящее элемент $r \in A$ в $\{r, s\}_{l}$. Очевидно, что любое такое дифференцирование равно нулю на $B$ и, следовательно, на любом алгебраическом расширении алгебры $B$. Значит, $\{B, A\}_{l}=0$. Отсюда аналогичным образом следует, что $\{A, A\}_{l}=0$. Таким образом, алгебра $\mathscr{A}$ коммутативна.

Пусть $G$ - группа Ли, $\mathfrak{g}=\operatorname{Lie} G$ и $U(\mathfrak{g})$ - универсальная обертывающая алгебра. На $U(\mathfrak{g})$ имеется естественная фильтрация, причем по теореме ПуанкареБиркгофа-Витта $\operatorname{gr} U(\mathfrak{g})=S(\mathfrak{g})=\mathbb{R}\left[\mathfrak{g}^{*}\right]$ - алгебра полиномов на $\mathfrak{g}^{*}$.

Отображение симметризации $\sigma: S(\mathfrak{g}) \rightarrow U(\mathfrak{g})$, характеризующееся свойством

$$
\sigma\left(\xi^{n}\right)=\xi^{n} \quad \forall \xi \in \mathfrak{g}, n \in \mathbb{Z}_{+},
$$

задает изоморфизм $G$-модулей $S(\mathfrak{g})$ и $U(\mathfrak{g})$.

На $S(\mathfrak{g})=\mathbb{R}\left[\mathfrak{g}^{*}\right]$ имеется скобка Пуассона-Ли $\{\cdot, \cdot\}$, которую можно определить двумя эквивалентными способами:

1) Пусть $\alpha \in \mathfrak{g}^{*}, f, h \in \mathbb{R}\left[\mathfrak{g}^{*}\right]$. Тогда $\{f, h\}(\alpha)=\left\langle\left[d_{\alpha} f, d_{\alpha} h\right], \alpha\right\rangle$.

2) Пусть $f \in S^{n}(\mathfrak{g}), h \in S^{m}(\mathfrak{g})$. Выберем $\tilde{f}, \tilde{h} \in U(\mathfrak{g})$ так, чтобы $\operatorname{gr}_{n} \tilde{f}=f$, $\operatorname{gr}_{m} \tilde{h}=h$. Тогда $\{f, h\}=\operatorname{gr}_{m+n-1}([\tilde{f}, \tilde{h}])$. (Легко видеть, что результат не зависит от выбора $\tilde{f}, \tilde{h}$. В частности, можно взять $\tilde{f}=\sigma(f), \tilde{h}=\sigma(h)$.)

Из определения 1) видно, что симплектическими слоями этой пуассоновой структуры являются $G$-орбиты в $\mathfrak{g}^{*}$, что позволяет задать пуассонову структуру на любом $G$-инвариантном подмногообразии в $\mathfrak{g}^{*}$.

Пусть $M-$ симплектическое многообразие и $G: M-$ пуассоново действие, т. е. задан такой гомоморфизм алгебр Ли $\mathfrak{g} \rightarrow C^{\infty}(M)$ (структура алгебры Ли на $C^{\infty}(M)$ задается скобкой Пуассона), $\xi \mapsto H_{\xi}$, что косой градиент функции $H_{\xi}$ 
равен $\xi_{*}$, где $\xi_{*}-$ поле скоростей действия, соответствующее элементу $\xi \in \mathfrak{g}$. В этом случае можно определить отображение моментов $\Phi: M \rightarrow \mathfrak{g}^{*}$ по формуле

$$
\langle\Phi(x), \xi\rangle=H_{\xi}(x) \quad\left(x \in T^{*} X, \xi \in \mathfrak{g}\right) .
$$

Это отображение является эквивариантным морфизмом пуассоновых многообразий, причем касательное пространство слоя в любой точке совпадает с ортогональным дополнением касательного пространства $G$-орбиты в этой точке относительно симплектической структуры на $M$.

Пусть $X=G / H-$ риманово однородное пространство (группа $H$ компактна). Рассмотрим подалгебру $H$-инвариантов в алгебре $\mathbb{R}\left[\mathfrak{h}^{\circ}\right]=\mathbb{R}\left[\mathfrak{g}^{*}\right] / \mathbb{R}\left[\mathfrak{g}^{*}\right] \mathfrak{h}$. Заметим, что

$$
P(X)^{G} \simeq \mathbb{R}\left[\mathfrak{h}^{\circ}\right]^{H}=\operatorname{gr} U(\mathfrak{g})^{H} /(U(\mathfrak{g}) \mathfrak{h})^{H} .
$$

Так как $\left(\mathbb{R}\left[\mathfrak{g}^{*}\right] \mathfrak{h}\right)^{H}$ - пуассонов идеал в $\mathbb{R}\left[\mathfrak{g}^{*}\right]^{H}$, то скобку Пуассона на $\mathbb{R}\left[\mathfrak{h}^{\circ}\right]^{H}$ можно корректно определить так: для $f, h \in \mathbb{R}\left[\mathfrak{h}^{\circ}\right]^{H}$ выберем такие $\tilde{f}, \tilde{h} \in \mathbb{R}\left[\mathfrak{g}^{*}\right]$, что $\left.\tilde{f}\right|_{\mathfrak{h}}{ }=f,\left.\tilde{h}\right|_{\mathfrak{h}}{ }=h$; тогда $\{f, h\}=\left.\{\tilde{f}, \tilde{h}\}\right|_{\mathfrak{h}}{ }^{\circ}$. Мы докажем, что можно аналогичным образом корректно определить скобку Пуассона на $\mathbb{R}\left[Y \cap \mathfrak{h}^{\circ}\right]^{H}$, где $Y-$ любое $G$-инвариантное замкнутое алгебраическое подмногообразие в $\mathfrak{g}^{*}$.

Рассмотрим отображение моментов $\Phi: T^{*} X \rightarrow \mathfrak{g}^{*}$. Образом этого отображения является множество $G \mathfrak{h}^{\circ} \subset \mathfrak{g}^{*}$ (кокасательное пространство в точке $g H \in X$ изоморфно отображается на подпространство $\left.\left(\mathrm{Ad}^{*} g\right) \mathfrak{h}^{\circ} \subset \mathfrak{g}^{*}\right)$. Прообраз инвариантного подмногообразия $Y \subset \mathfrak{g}^{*}$, очевидно, равен $G \Phi^{-1}\left(Y \cap \mathfrak{h}^{\circ}\right)$. Пусть $I \subset P(X)^{G}$ - идеал функций, обращающихся в нуль на $\Phi^{-1}(Y)$. Косой градиент любой функции из $I$ в любой точке $x \in \Phi^{-1}(Y)$ ортогонален слою отображения моментов, а значит, касается многообразия $G x$. Но косые градиенты $G$-инвариантных функций в точке $x$ ортогональны $G x$; следовательно, $\left\{P(X)^{G}, I\right\} \subset I$. Таким образом, $I$ - пуассонов идеал в $P(X)^{G}$, а значит, скобка Пуассона корректно определена на алгебре $\left.P(X)^{G}\right|_{\Phi^{-1}(Y)}$. Но ограничение на $\left(T_{o}^{*} X\right) \cap \Phi^{-1}(Y)=Y \cap \mathfrak{h}^{\circ}$ задает изоморфизм

$$
\left.P(X)^{G}\right|_{\Phi^{-1}(Y)} \simeq \mathbb{R}\left[Y \cap \mathfrak{h}^{\circ}\right]^{H}
$$

Таким образом, скобку Пуассона можно корректно определить на алгебре $\mathbb{R}\left[Y \cap \mathfrak{h}^{\circ}\right]^{H}$.

ЛЕмма 2. Пусть $G$ - алгебраическая группа и $H-$ компактная подгруппа в $G$. Пусть $Y$ есть $G$-инвариантное замкнутое подмногообразие в $\mathfrak{g}^{*}$, причем алгебра $\mathbb{R}\left[Y \cap \mathfrak{h}^{\circ}\right]^{H}$ коммутативна относительно скобки Пуассона. Тогда для точки общего положения $y \in \mathfrak{h}^{\circ} \cap Y$ многообразие Gy $\mathfrak{h}^{\circ}$ является конечным объединением $H$-орбит.

ДокАЗАТЕЛьство. Пусть $x$ - точка общего положения в $G y \cap \mathfrak{h}^{\circ}$. Очевидно, что пространство $\left(\mathrm{ad}^{*} \mathfrak{h}\right) x$ изотропно, а пространство $\left(\mathrm{ad}^{*} \mathfrak{g}\right) x \cap \mathfrak{h}^{\circ}$ является его ортогональным дополнением относительно формы Кириллова на Gy. Таким образом, пространство $\left(\left(\mathrm{ad}^{*} \mathfrak{g}\right) x \cap \mathfrak{h}^{\circ}\right) /\left(\mathrm{ad}^{*} \mathfrak{h}\right) x$ симплектично. Так как $H$-орбиты в $G y \cap \mathfrak{h}^{\circ}$ разделяются полиномиальными инвариантами, то косые градиенты $H$-инвариантов в точке $x$ заполняют пространство $\left(\left(\operatorname{ad}^{*} \mathfrak{g}\right) x \cap \mathfrak{h}^{\circ}\right) /\left(\mathrm{ad}^{*} \mathfrak{h}\right) x$. Но по условию эти градиенты должны коммутировать. Следовательно, пространство $\left(\left(\mathrm{ad}^{*} \mathfrak{g}\right) x \cap \mathfrak{h}^{\circ}\right) /\left(\mathrm{ad}^{*} \mathfrak{h}\right) x$ нулевое. Таким образом, $H$-орбиты общего положения открыты в $G y \cap \mathfrak{h}^{\circ}$. Так как группа $H$ компактна, то они и замкнуты, откуда следует утверждение леммы. 
Лемма 3. Пусть $G$ - редуктивная группа, а $Y$ есть $G$-инвариантное замкнутое подмногообразие в $\mathfrak{g}^{*}$. Тогда $\mathbb{R}(Y)^{G} \supseteq \operatorname{Quot}\left(\left.\mathbb{R}\left[\mathfrak{g}^{*}\right]^{G}\right|_{Y}\right)$ - конечное расширение полей.

ДокАЗАТЕЛЬСтво. Каждый слой морфизма факторизации $\mathfrak{g}^{*} \rightarrow \mathfrak{g}^{*} / / G$ является объединением конечного числа орбит. Значит, то же самое верно для морфизма $Y \rightarrow Y / / G$. Следовательно, степени трансцендентности полей $\mathbb{R}(Y)^{G}$ и $\operatorname{Quot}\left(\mathbb{R}[Y]^{G}\right)$ равны, т. е. $\mathbb{R}(Y)^{G} \supseteq \operatorname{Quot}\left(\mathbb{R}[Y]^{G}\right)$ - конечное расширение. Но так как группа $G$ редуктивна, то $\operatorname{Quot}\left(\mathbb{R}[Y]^{G}\right)=\operatorname{Quot}\left(\left.\mathbb{R}\left[\mathfrak{g}^{*}\right]^{G}\right|_{Y}\right)$. Отсюда следует утверждение леммы.

Пусть $G=G_{0} \wedge G_{1}$, где группы $G_{0}$ и $G_{1}$ редуктивны, и $H-$ компактная подгруппа в $G_{0}$. Пусть $J_{1}-$ такой $G_{0}$-инвариантный двусторонний идеал в $U\left(\mathfrak{g}_{1}\right)$, что $I_{1}=\operatorname{gr} J_{1}-$ простой идеал в $S\left(\mathfrak{g}_{1}\right)$. Тогда $J=U(\mathfrak{g}) J_{1}=U\left(\mathfrak{g}_{0}\right) J_{1}-$ двусторонний идеал в $U(\mathfrak{g})$, а $I=\operatorname{gr} J=S\left(\mathfrak{g}_{0}\right) I_{1}$ есть $G$-инвариантный идеал в $S(\mathfrak{g})$. Имеют место следующие изоморфизмы $H$-модулей:

$J+U(\mathfrak{g}) \mathfrak{h} \simeq\left(\left[U\left(\mathfrak{g}_{0}\right) / U\left(\mathfrak{g}_{0}\right) \mathfrak{h}\right] \otimes J_{1}\right) \oplus U(\mathfrak{g}) \mathfrak{h} \simeq\left(S\left(\mathfrak{g}_{0} / \mathfrak{h}\right) \otimes I_{1}\right) \oplus S(\mathfrak{g}) \mathfrak{h} \simeq I+S(\mathfrak{g}) \mathfrak{h}$.

Отсюда следует, что

$$
\operatorname{gr}(J+U(\mathfrak{g}) \mathfrak{h})^{H} \simeq(I+S(\mathfrak{g}) \mathfrak{h})^{H},
$$

а значит, имеет место изоморфизм пуассоновых алгебр:

$$
\operatorname{gr} U(\mathfrak{g})^{H} /(J+U(\mathfrak{g}) \mathfrak{h})^{H} \simeq S(\mathfrak{g})^{H} /(I+S(\mathfrak{g}) \mathfrak{h})^{H} .
$$

Лемма 4. Коммутативность пуассоновой алгебры $S(\mathfrak{g})^{H} /(I+S(\mathfrak{g}) \mathfrak{h})^{H}$ влечет за собой коммутативность ассоцииативной алгебры $U(\mathfrak{g})^{H} /(J+U(\mathfrak{g}) \mathfrak{h})^{H}$.

ДокАЗАТЕЛЬСтво. Пусть $Y \subset \mathfrak{g}^{*}-$ многообразие нулей идеала $I$ и $Y_{1} \subset \mathfrak{g}_{1}^{*}-$ многообразие нулей идеала $I_{1}$. Заметим, что $Y=Y_{1} \times \mathfrak{g}_{0}^{*}$, причем имеют место следующие изоморфизмы алгебр:

$$
\mathbb{R}\left[Y \cap \mathfrak{h}^{\circ}\right]^{H} \simeq\left(\left(S\left(\mathfrak{g}_{1}\right) / I_{1}\right) \otimes\left(S\left(\mathfrak{g}_{0}\right) / S\left(\mathfrak{g}_{0}\right) \mathfrak{h}\right)\right)^{H} \simeq S(\mathfrak{g})^{H} /(I+S(\mathfrak{g}) \mathfrak{h})^{H} .
$$

По лемме 2 каждая $G$-орбита общего положения в $Y$ пересекает $\mathfrak{h}^{\circ}$ не более чем по конечному числу $H$-орбит. Таким образом, поле $\mathbb{R}\left(Y \cap \mathfrak{h}^{\circ}\right)^{H}$ является конечным расширением поля $\left.\mathbb{R}(Y)^{G}\right|_{Y \cap \mathfrak{h}^{\circ}}$. Так как группа $G$ редуктивна, то по лемме 3 поле $\mathbb{R}\left(Y \cap \mathfrak{h}^{\circ}\right)^{H}$ является конечным расширением поля $\operatorname{Quot}\left(\left.\mathbb{R}\left[\mathfrak{g}^{*}\right]^{G}\right|_{Y \cap \mathfrak{h}^{\circ}}\right)$. Но ограничения всех инвариантов из $\mathbb{R}\left[\mathfrak{g}^{*}\right]^{G}$ на $Y \cap \mathfrak{h}^{\circ}$ принадлежат $\operatorname{gr} Z\left(U(\mathfrak{g})^{H} /(J+U(\mathfrak{g}) \mathfrak{h})^{H}\right)$. Значит, алгебра $S(\mathfrak{g})^{H} /(I+S(\mathfrak{g}) \mathfrak{h})^{H}$ является алгебраическим расширением алгебры $\operatorname{gr} Z\left(U(\mathfrak{g})^{H} /(J+U(\mathfrak{g}) \mathfrak{h})^{H}\right)$. По лемме 1 отсюда следует коммутативность алгебры $U(\mathfrak{g})^{H} /(J+U(\mathfrak{g}) \mathfrak{h})^{H}$.

Пусть $\mathfrak{a}$ - векторное пространство с постоянной (возможно, вырожденной) кососимметрической билинейной формой $\omega$. Пусть $W(\mathfrak{a})-$ алгебра Вейля с пространством образующих $\mathfrak{a}$ и определяющими соотношениями

$$
v w-w v=\omega(v, w)
$$

для любых $v, w \in \mathfrak{a}$.

На алгебре $W(\mathfrak{a})$ имеется фильтрация по степени выражения через образующие:

$$
W(\mathfrak{a})=\bigcup_{n=0}^{\infty} W^{(n)}(\mathfrak{a}),
$$


причем ассоциированная градуированная алгебра изоморфна $S(\mathfrak{a})$.

Отображение симметризации $\sigma: S(\mathfrak{a}) \rightarrow W(\mathfrak{a})$, определяемое так же, как и для универсальной обертывающей алгебры, свойством

$$
\sigma\left(\xi^{n}\right)=\xi^{n} \quad \forall \xi \in \mathfrak{a}, n \in \mathbb{Z}_{+},
$$

задает изоморфизм $S p(\mathfrak{a}, \omega)$-модулей $S(\mathfrak{a})$ и $W(\mathfrak{a})$.

$\mathrm{Ha} \mathbb{R}\left[\mathfrak{a}^{*}\right]=S(\mathfrak{a})$ имеется скобка Пуассона

$$
\{\cdot, \cdot\}: S^{n}(\mathfrak{a}) \otimes S^{m}(\mathfrak{a}) \rightarrow S^{n+m-2}(\mathfrak{a}),
$$

определяемая коммутатором в $W(\mathfrak{a})$. Если форма $\omega$ невырожденна, то эта скобка совпадает со скобкой Пуассона, определяемой постоянной симплектической структурой на $\mathfrak{a}$.

Пусть $G$ - группа Ли, действующая на пространстве $\mathfrak{a}$ преобразованиями, сохраняющими форму $\omega$. Определим «полупрямое тензорное произведение» $U(\mathfrak{g}) \otimes W(\mathfrak{a}):$ это произведение алгебр $U(\mathfrak{g})$ и $W(\mathfrak{a})$ с определяющими соотношениями

$$
x a-a x=x(a) \quad \forall x \in \mathfrak{g}, a \in \mathfrak{a} .
$$

Фильтрации на $U(\mathfrak{g})$ и $W(\mathfrak{a})$ индуцируют фильтрацию на $U(\mathfrak{g}) \otimes W(\mathfrak{a})$ :

$$
(U(\mathfrak{g}) \otimes W(\mathfrak{a}))^{(n)}=\sum_{2 m+k=n} U^{(m)}(\mathfrak{g}) \otimes W^{(k)}(\mathfrak{a}),
$$

т. е. пространство $\mathfrak{g}$ имеет вес 2 , а $\mathfrak{a}-$ вес 1 . Ассоциированная градуированная алгебра изоморфна $S(\mathfrak{g}) \otimes S(\mathfrak{a})=\mathbb{R}\left[\mathfrak{g}^{*} \times \mathfrak{a}^{*}\right]$. Коммутатор в алгебре $U(\mathfrak{g}) \otimes W(\mathfrak{a})$ определяет скобку Пуассона на $S(\mathfrak{g}) \otimes S(\mathfrak{a})$ :

$$
\begin{aligned}
\{\cdot, \cdot\}:\left(S^{n_{1}}(\mathfrak{g}) \otimes S^{m_{1}}(\mathfrak{a})\right) \otimes\left(S^{n_{2}}(\mathfrak{g}) \otimes S^{m_{2}}(\mathfrak{a})\right) \\
\quad \rightarrow\left(S^{n_{1}+n_{2}}(\mathfrak{g}) \otimes S^{m_{1}+m_{2}-2}(\mathfrak{a})\right) \oplus\left(S^{n_{1}+n_{2}-1}(\mathfrak{g}) \otimes S^{m_{1}+m_{2}}(\mathfrak{a})\right) .
\end{aligned}
$$

Пусть $H-$ компактная подгруппа в $G$. Рассмотрим подалгебру $H$-инвариантов в алгебре $U(\mathfrak{g}) \otimes W(\mathfrak{a})$. Очевидно, что $(U(\mathfrak{g}) \mathfrak{h} \otimes W(\mathfrak{a}))^{H}-$ двусторонний идеал в $U(\mathfrak{g}) \bigotimes W(\mathfrak{a}))^{H}$. Рассмотрим следующую ассоциативную алгебру:

$$
B(\mathfrak{g}, \mathfrak{h}, \mathfrak{a})=(U(\mathfrak{g}) \otimes W(\mathfrak{a}))^{H} /(U(\mathfrak{g}) \mathfrak{h} \otimes W(\mathfrak{a}))^{H} .
$$

Фильтрация на $U(\mathfrak{g}) \otimes W(\mathfrak{a})$ задает фильтрацию на $B(\mathfrak{g}, \mathfrak{h}, \mathfrak{a})$, причем ассоциированная градуированная (пуассонова) алгебра изоморфна $(S(\mathfrak{g} / \mathfrak{h}) \otimes S(\mathfrak{a}))^{H}=$ $\mathbb{R}\left[\mathfrak{h}^{\circ} \times \mathfrak{a}^{*}\right]^{H}$.

Теорема 1. Пусть $G$ - редуктивная группа, действующая симплектическими преобразованиями на пространстве а с невырожденной 2-формой $\omega, u$ $H$ - компактная подгруппа в $G$. Тогда коммутативность алгебрь $\mathbb{R}\left[\mathfrak{h}^{\circ} \times \mathfrak{a}^{*}\right]^{H}$ относительно скобки Пуассона влечет за собой коммутативность алгебрь $B(\mathfrak{g}, \mathfrak{h}, \mathfrak{a})$.

ДокАЗАТЕЛьство. На $W(\mathfrak{a})$ имеется естественная $\mathbb{Z}_{2}$-градуировка $W(\mathfrak{a})=$ $W(\mathfrak{a})_{+} \oplus W(\mathfrak{a})_{-}$по степеням одночленов. Соответствующая градуировка имеется на $\mathbb{R}\left[\mathfrak{a}^{*}\right], B(\mathfrak{g}, \mathfrak{h}, \mathfrak{a})$ и $\mathbb{R}\left[\mathfrak{h}^{\circ} \times \mathfrak{a}^{*}\right]^{H}$.

Заметим, что скобка Пуассона задает на $S^{2}(\mathfrak{a})$ структуру алгебры Ли, изоморфной $\mathfrak{s p}(\mathfrak{a}, \omega)$. Рассмотрим пространство $W(\mathfrak{a})_{2}=\sigma\left(S^{2}(\mathfrak{a})\right) \subset W(\mathfrak{a})$. Коммутатор задает на $W(\mathfrak{a})_{2}$ структуру алгебры Ли, изоморфной $\mathfrak{s p}(\mathfrak{a}, \omega)$. Так как пространство $W(\mathfrak{a})_{2}$ порождает всю алгебру $W(\mathfrak{a})_{+}$, то алгебра $W(\mathfrak{a})_{+}$изоморфна 
факторалгебре алгебры $U(\mathfrak{s p}(\mathfrak{a}, \omega))$ по некоторому $G$-инвариантному двустороннему идеалу $J_{1}$.

На пространстве $\mathfrak{g}+W(\mathfrak{a})_{2}$ коммутатор задает структуру алгебры Ли, изоморфной $\mathfrak{g}(+\mathfrak{s} \mathfrak{p}(\mathfrak{a}, \omega)$ (на самом деле полупрямая сумма алгебр $\mathfrak{g}$ и $\mathfrak{s p}(\mathfrak{a}, \omega)$ изоморфна прямой сумме, но для дальнейших рассмотрений это не важно). Значит, алгебра $U(\mathfrak{g}) \otimes W(\mathfrak{a})_{+}$изоморфна факторалгебре алгебры $U(\mathfrak{g}+\mathfrak{s} \mathfrak{p}(\mathfrak{a}, \omega))$ по идеалу $J=U(\mathfrak{g}) J_{1}$.

Рассмотрим $(G<S p(\mathfrak{a}, \omega))$-инвариантный пуассонов идеал $I_{1}=\operatorname{gr} J_{1}$ в пуассоновой алгебре $S(\mathfrak{s p}(\mathfrak{a}, \omega))=\mathbb{R}\left[\mathfrak{s p}(\mathfrak{a}, \omega)^{*}\right]$. Имеют место следующие изоморфизмы градуированных алгебр:

$$
S(\mathfrak{s p}(\mathfrak{a}, \omega)) / I_{1}=\operatorname{gr} U(\mathfrak{s p}(\mathfrak{a}, \omega)) / J_{1}=\mathbb{R}\left[\mathfrak{a}^{*}\right]_{+} .
$$

Отсюда вытекает, что алгебра $S(\mathfrak{s p}(\mathfrak{a}, \omega)) / I_{1}$ не содержит делителей нуля, т. е. идеал $I_{1}=$ gr $J_{1}$ прост.

Подалгебра $H$-инвариантов в $\operatorname{gr} U\left(\mathfrak{g}(+\mathfrak{s p}(\mathfrak{a}, \omega)) / J=\mathbb{R}\left[\mathfrak{h}^{\circ} \times \mathfrak{a}^{*}\right]_{+}\right.$коммутативна относительно скобки Пуассона. Полагая в лемме $4 G_{0}=G, G_{1}=S p(\mathfrak{a}, \omega)$, получаем, что алгебра $B_{+}(\mathfrak{g}, \mathfrak{h}, \mathfrak{a})$ коммутативна. По лемме 1 и сама алгебра $B(\mathfrak{g}, \mathfrak{h}, \mathfrak{a})$ коммутативна.

\section{§4. Доказательство основного результата}

Предлагаемое рассуждение является модификацией доказательства коммутативности слабо коммутативных пространств гейзенбергова типа, изложенного в [1].

Теорема 2. Пусть $X=G / H-$ риманово однородное пространство. Тогда коммутативность пространства $X$ равносильна его слабой коммутативности.

ДокАЗАТЕЛЬСтво. Пусть пространство $X$ слабо коммутативно. Известно [1], что в этом случае $G=L \curlywedge N$, где

1) $N$ - не более чем 2 -ступенно нильпотентная группа Ли;

2) $L-$ редуктивная подгруппа Ли, содержащая $H$;

3) $\mathbb{R}[\mathfrak{n}]^{L}=\mathbb{R}[\mathfrak{n}]^{H}$.

Пусть $\mathfrak{z}-$ коммутант алгебры Ли $\mathfrak{n}$ (содержащийся в ее центре) и $\mathfrak{v}-$ какоелибо $L$-инвариантное дополнительное подпространство. Для любой линейной функции $\alpha \in \mathfrak{z}^{*}$ обозначим через $\hat{\alpha}$ кососимметрическую билинейную функцию на $\mathfrak{v}$, определяемую по формуле

$$
\hat{\alpha}(\xi, \eta)=\alpha([\xi, \eta]) .
$$

Пусть $L_{\alpha}$ и $H_{\alpha}-$ стабилизаторы функции $\alpha$ в группах $L$ и $H$ соответственно. Из условия 3) следует, что фактор группы $L$ по ядру неэффективности действия $L: \mathfrak{n}^{*}$ компактен. Следовательно, группа $L_{\alpha}$ редуктивна. Подпространство $\operatorname{Ker} \hat{\alpha} \subset \mathfrak{v}$, очевидно, $L_{\alpha}$-инвариантно. Пусть $\mathfrak{v}_{\alpha}-$ какое-либо $L_{\alpha}$-инвариантное дополнительное пространство. Естественное линейное представление группы $L_{\alpha}$ (и $H_{\alpha}$ ) в симплектическом векторном пространстве $\mathfrak{v}_{\alpha} \simeq \mathfrak{v} / \operatorname{Ker} \hat{\alpha}$ является симплектическим.

Для любой линейной функции $\alpha \in \mathfrak{z}^{*}$ имеется очевидный гомоморфизм

$$
\varphi_{\alpha}: U(\mathfrak{n}) \rightarrow W_{\alpha}(\mathfrak{v})=U(\mathfrak{n}) /(\xi-\alpha(\xi): \xi \in \mathfrak{z}),
$$


образ которого представляет собой алгебру Вейля (возможно, вырожденную), определяемую формой $\hat{\alpha}$ на пространстве $\mathfrak{v}$.

Для любой линейной функции $\beta \in(\operatorname{Ker} \hat{\alpha})^{*}$ имеется гомоморфизм

$$
\psi_{\alpha, \beta}: W_{\alpha}(\mathfrak{v}) \rightarrow W_{\alpha, \beta}\left(\mathfrak{v}_{\alpha}\right)=W_{\alpha}(\mathfrak{v}) /(\xi-\beta(\xi): \xi \in \operatorname{Ker} \hat{\alpha}),
$$

образом которого является уже невырожденная алгебра Вейля.

Заметим, что имеется естественная $L$-эквивариантная биекция между множеством пар $\left(\alpha \in \mathfrak{z}^{*}, \beta \in(\operatorname{Ker} \hat{\alpha})^{*}\right)$ и множеством $N$-орбит в $\mathfrak{n}^{*}$. А именно, пусть $p: \mathfrak{n}^{*} \rightarrow \mathfrak{z}^{*}-$ естественная проекция, двойственная вложению $\mathfrak{z} \rightarrow \mathfrak{n}$, а $p_{\alpha}: \mathfrak{n}^{*} \rightarrow(\operatorname{Ker} \hat{\alpha})^{*}-$ естественная проекция, двойственная вложению Ker $\hat{\alpha} \rightarrow \mathfrak{n}$. Аффинное пространство $O_{\alpha, \beta}=p^{-1}(\alpha) \cap p_{\alpha}^{-1}(\beta)$, очевидно, $N$-инвариантно, причем скобка Пуассона-Ли на нем невырожденна. Так как симплектическими слоями пуассоновой структуры на $\mathfrak{n}^{*}$ являются $N$-орбиты в $\mathfrak{n}^{*}$, то пространство $O_{\alpha, \beta}$ содержит открытую $N$-орбиту. Но группа $N$ унипотентна, а значит, все $N$-орбиты в $\mathfrak{n}^{*}$ замкнуты; следовательно, пространство $O_{\alpha, \beta}$ является $N$-орбитой. Таким образом, каждой паре $\left(\alpha \in \mathfrak{z}^{*}, \beta \in(\operatorname{Ker} \hat{\alpha})^{*}\right)$ соответствует орбита. С другой стороны, если $O=N x$ - орбита в $\mathfrak{n}^{*}$, то $O=O_{p(x), p_{p(x)}(x)}$; следовательно, построенное отображение $(\alpha, \beta) \mapsto O_{\alpha, \beta}$ биективно.

Из этой конструкции видно, что гомоморфизм пуассоновых алгебр

$$
\operatorname{gr}\left(\psi_{\alpha, \beta} \circ \varphi_{\alpha}\right): \mathbb{R}\left[\mathfrak{n}^{*}\right]=S(\mathfrak{n}) \rightarrow S\left(\mathfrak{v}_{\alpha}\right)=\operatorname{gr} W_{\alpha, \beta}\left(\mathfrak{v}_{\alpha}\right)
$$

имеет очевидную геометрическую интерпретацию: это гомоморфизм ограничения на орбиту $O_{\alpha, \beta} \subset \mathfrak{n}^{*}$, являющуюся аффинным подпространством в $\mathfrak{n}^{*}$.

Пусть $H_{\alpha, \beta}$ и $L_{\alpha, \beta}$ - стабилизаторы орбиты $O_{\alpha, \beta}$ в $H_{\alpha}$ и $L_{\alpha}$ соответственно (или, что то же самое, стабилизаторы функционала $\beta$ в $H_{\alpha}$ и $L_{\alpha}$ ). Так как фактор группы $L$ по ядру неэффективности действия $L: \mathfrak{n}^{*}$ компактен, то группа $L_{\alpha, \beta}$ редуктивна.

Мы докажем, что для пары $(\alpha, \beta)$ общего положения сужение гомоморфизма

$$
\psi_{\alpha, \beta} \circ \varphi_{\alpha}: U(\mathfrak{n}) \rightarrow W_{\alpha, \beta}\left(\mathfrak{v}_{\alpha}\right)
$$

на $U(\mathfrak{n})^{H}$ продолжается до гомоморфизма

$$
U(\mathfrak{g})^{H} /(U(\mathfrak{g}) \mathfrak{h})^{H} \rightarrow B\left(\mathfrak{l}_{\alpha, \beta}, \mathfrak{h}_{\alpha, \beta}, \mathfrak{v}_{\alpha}\right) .
$$

Из условия 3) следует, что для точки $\gamma \in \mathfrak{n}^{*}$ общего положения

$$
\mathfrak{l}=\mathfrak{h}+\mathfrak{l}_{\gamma},
$$

а значит, для пары $(\alpha, \beta)$ общего положения

$$
\mathfrak{l}=\mathfrak{h}+\mathfrak{l}_{\alpha}=\mathfrak{h}+\mathfrak{l}_{\alpha, \beta} .
$$

Отсюда следует, что для любого класса $U(\mathfrak{g}) / U(\mathfrak{g}) \mathfrak{h}$ можно выбрать представитель в $U\left(\mathfrak{l}_{\alpha}(\mathfrak{n})\right.$. Этот представитель определяется с точностью до $U(\mathfrak{g}) \mathfrak{h} \cap$ $U\left(\mathfrak{l}_{\alpha}(+\mathfrak{n})=U\left(\mathfrak{l}_{\alpha}(+\mathfrak{n}) \mathfrak{h}_{\alpha}\right.\right.$. Значит, имеется изоморфизм фильтрованных векторных пространств

$$
U(\mathfrak{g}) / U(\mathfrak{g}) \mathfrak{h} \simeq U\left(\mathfrak{l}_{\alpha}(+\mathfrak{n}) / U\left(\mathfrak{l}_{\alpha}(+\mathfrak{n}) \mathfrak{h}_{\alpha} .\right.\right.
$$

Очевидно, что этот изоморфизм $H_{\alpha}$-эквивариантен. Таким образом, для точки $\alpha \in \mathfrak{z}^{*}$ общего положения имеется естественное вложение ассоциативных алгебр

$$
\begin{aligned}
i_{\alpha}: U(\mathfrak{g})^{H} /(U(\mathfrak{g}) \mathfrak{h})^{H}=U(\mathfrak{l}+\mathfrak{n})^{H} /(U(\mathfrak{l}+\mathfrak{n}) \mathfrak{h})^{H} \\
\quad \hookrightarrow U\left(\mathfrak{l}(\mathfrak{n})^{H_{\alpha}} /(U(\mathfrak{l}+\mathfrak{n}) \mathfrak{h})^{H_{\alpha}}=U\left(\mathfrak{l}_{\alpha}(+\mathfrak{n})^{H_{\alpha}} /\left(U\left(\mathfrak{l}_{\alpha}(+\mathfrak{n}) \mathfrak{h}_{\alpha}\right)^{H_{\alpha}} .\right.\right.\right.
\end{aligned}
$$


Гомоморфизм $\varphi_{\alpha}$, очевидно, $L_{\alpha}$-эквивариантен, а значит, он индуцирует гомоморфизм ассоциативных алгебр

$$
\begin{gathered}
\Phi_{\alpha}: U\left(\mathfrak{l}_{\alpha}(+\mathfrak{n})^{H_{\alpha}} /\left(U\left(\mathfrak{l}_{\alpha}(+\mathfrak{n}) \mathfrak{h}_{\alpha}\right)^{H_{\alpha}}=\left(U\left(\mathfrak{l}_{\alpha}\right) \otimes U(\mathfrak{n})\right)^{H_{\alpha}} /\left(U\left(\mathfrak{l}_{\alpha}\right) \mathfrak{h}_{\alpha} \otimes U(\mathfrak{n})\right)^{H_{\alpha}}\right.\right. \\
\rightarrow\left(U\left(\mathfrak{l}_{\alpha}\right) \otimes W_{\alpha}(\mathfrak{v})\right)^{H_{\alpha}} /\left(U\left(\mathfrak{l}_{\alpha}\right) \mathfrak{h}_{\alpha} \otimes W_{\alpha}(\mathfrak{v})\right)^{H_{\alpha}}=B\left(\mathfrak{l}_{\alpha}, \mathfrak{h}_{\alpha}, \mathfrak{v}\right) .
\end{gathered}
$$

Аналогично, для пары $(\alpha, \beta)$ общего положения имеется $H_{\alpha, \beta}$-эквивариантный изоморфизм фильтрованных векторных пространств

$$
U\left(\mathfrak{l}_{\alpha}\right) / U\left(\mathfrak{l}_{\alpha}\right) \mathfrak{h}_{\alpha} \simeq U\left(\mathfrak{l}_{\alpha, \beta}\right) / U\left(\mathfrak{l}_{\alpha, \beta}\right) \mathfrak{h}_{\alpha, \beta},
$$

а значит, и

$$
U\left(\mathfrak{l}_{\alpha}\right) \otimes W_{\alpha}(\mathfrak{v}) / U\left(\mathfrak{l}_{\alpha}\right) \mathfrak{h}_{\alpha} \otimes W_{\alpha}(\mathfrak{v}) \simeq U\left(\mathfrak{l}_{\alpha, \beta}\right) \otimes W_{\alpha}(\mathfrak{v}) / U\left(\mathfrak{l}_{\alpha, \beta}\right) \mathfrak{h}_{\alpha, \beta} \otimes W_{\alpha}(\mathfrak{v}) .
$$

Таким образом, имеется естественное вложение ассоциативных алгебр

$$
\begin{array}{r}
i_{\alpha, \beta}: B\left(\mathfrak{l}_{\alpha}, \mathfrak{h}_{\alpha}, \mathfrak{v}\right)=\left(U\left(\mathfrak{l}_{\alpha}\right) \otimes W_{\alpha}(\mathfrak{v})\right)^{H_{\alpha}} /\left(U\left(\mathfrak{l}_{\alpha}\right) \mathfrak{h}_{\alpha} \otimes W_{\alpha}(\mathfrak{v})\right)^{H_{\alpha}} \\
\hookrightarrow\left(U\left(\mathfrak{l}_{\alpha, \beta}\right) \otimes W_{\alpha}(\mathfrak{v})\right)^{H_{\alpha, \beta}} /\left(U\left(\mathfrak{l}_{\alpha, \beta}\right) \mathfrak{h}_{\alpha, \beta} \otimes W_{\alpha}(\mathfrak{v})\right)^{H_{\alpha, \beta}} .
\end{array}
$$

Гомоморфизм $\psi_{\alpha, \beta}$, очевидно, $L_{\alpha, \beta}$-эквивариантен, а значит, он индуцирует гомоморфизм ассоциативных алгебр

$$
\begin{aligned}
& \Psi_{\alpha, \beta}:\left(U\left(\mathfrak{l}_{\alpha, \beta}\right) \otimes W_{\alpha}(\mathfrak{v})\right)^{H_{\alpha, \beta}} /\left(U\left(\mathfrak{l}_{\alpha, \beta}\right) \mathfrak{h}_{\alpha, \beta} \otimes W_{\alpha}(\mathfrak{v})\right)^{H_{\alpha, \beta}} \\
& \rightarrow\left(U\left(\mathfrak{l}_{\alpha, \beta}\right) \otimes W_{\alpha, \beta}\left(\mathfrak{v}_{\alpha}\right)\right)^{H_{\alpha, \beta}} /\left(U\left(\mathfrak{l}_{\alpha, \beta}\right) \mathfrak{h}_{\alpha, \beta} \otimes W_{\alpha, \beta}\left(\mathfrak{v}_{\alpha}\right)\right)^{H_{\alpha, \beta}}=B\left(\mathfrak{l}_{\alpha, \beta}, \mathfrak{h}_{\alpha, \beta}, \mathfrak{v}_{\alpha}\right) .
\end{aligned}
$$

Рассмотрим гомоморфизм

$$
\Psi_{\alpha, \beta} \circ i_{\alpha, \beta} \circ \Phi_{\alpha} \circ i_{\alpha}: U(\mathfrak{g})^{H} /(U(\mathfrak{g}) \mathfrak{h})^{H} \rightarrow B\left(\mathfrak{l}_{\alpha, \beta}, \mathfrak{h}_{\alpha, \beta}, \mathfrak{v}_{\alpha}\right) .
$$

Гомоморфизм ассоциированных пуассоновых алгебр

$$
\begin{aligned}
& \operatorname{gr}\left(\Psi_{\alpha, \beta} \circ i_{\alpha, \beta} \circ \Phi_{\alpha} \circ i_{\alpha}\right): \mathbb{R}\left[\mathfrak{h}^{\circ}\right]^{H}=S(\mathfrak{g})^{H} /(S(\mathfrak{g}) \mathfrak{h})^{H} \\
\rightarrow & \left(S\left(\mathfrak{l}_{\alpha, \beta} / \mathfrak{h}_{\alpha, \beta}\right) \otimes S\left(\mathfrak{v}_{\alpha}\right)\right)^{H_{\alpha, \beta}}=\mathbb{R}\left[\left(\mathfrak{l}_{\alpha, \beta} / \mathfrak{h}_{\alpha, \beta}\right)^{*} \times O_{\alpha, \beta}\right]^{H_{\alpha, \beta}}=\mathbb{R}\left[(\mathfrak{l} / \mathfrak{h})^{*} \times O_{\alpha, \beta}\right]^{H_{\alpha, \beta}}
\end{aligned}
$$

является гомоморфизмом ограничения на замкнутое алгебраическое подмногообразие $(\mathfrak{l} / \mathfrak{h})^{*} \times O_{\alpha, \beta} \subset \mathfrak{h}^{\circ}$. Заметим, что $H_{\alpha, \beta}$-орбиты в этом подмногообразии суть пересечения $H$-орбит в $\mathfrak{h}^{\circ}$ с подмногообразием $(\mathfrak{l} / \mathfrak{h})^{*} \times O_{\alpha, \beta} \subset \mathfrak{h}^{\circ}$. Это означает, что любой $H_{\alpha, \beta}$-инвариантный полином на $(\mathfrak{l} / \mathfrak{h})^{*} \times O_{\alpha, \beta}$ является ограничением некоторого $H$-инвариантного полинома на $\mathfrak{h}^{\circ}$. Таким образом, гомоморфизм $\operatorname{gr}\left(\Psi_{\alpha, \beta} \circ i_{\alpha, \beta} \circ \Phi_{\alpha} \circ i_{\alpha}\right)$ сюръективен, а значит, пуассонова алгебpa $\left(S\left(\mathfrak{l}_{\alpha, \beta} / \mathfrak{h}_{\alpha, \beta}\right) \otimes S\left(\mathfrak{v}_{\alpha}\right)\right)^{H_{\alpha, \beta}}=\operatorname{gr} B\left(\mathfrak{l}_{\alpha, \beta}, \mathfrak{h}_{\alpha, \beta}, \mathfrak{v}_{\alpha}\right)$ коммутативна. По теореме 1 ассоциативная алгебра $B\left(\mathfrak{l}_{\alpha, \beta}, \mathfrak{h}_{\alpha, \beta}, \mathfrak{v}_{\alpha}\right)$ коммутативна.

Алгебру $D(X)^{G} \simeq U(\mathfrak{g})^{H} /(U(\mathfrak{g}) \mathfrak{h})^{H}$ можно рассматривать как алгебру сечений расслоения над $\operatorname{Spec} S(\mathfrak{z})=\mathfrak{z}^{*}$, слой которого над точкой $\alpha \in \mathfrak{z}^{*}$ есть алгебра

$$
\Phi_{\alpha} \circ i_{\alpha}\left(U(\mathfrak{g})^{H} /(U(\mathfrak{g}) \mathfrak{h})^{H}\right)=B\left(\mathfrak{l}_{\alpha}, \mathfrak{h}_{\alpha}, \mathfrak{v}\right) .
$$

Таким образом, достаточно доказать коммутативность алгебры $B\left(\mathfrak{l}_{\alpha}, \mathfrak{h}_{\alpha}, \mathfrak{v}\right)$ для любой точки $\alpha \in \mathfrak{z}^{*}$ общего положения.

В свою очередь, если форма $\hat{\alpha}$ вырожденна, алгебру $B\left(\mathfrak{l}_{\alpha}, \mathfrak{h}_{\alpha}, \mathfrak{v}\right)$ можно рассматривать как алгебру сечений расслоения над 
слой которого над точкой $\beta \in(\operatorname{Ker} \hat{\alpha})^{*}$ есть алгебра

$$
\Psi_{\alpha, \beta} \circ i_{\alpha, \beta}\left(B\left(\mathfrak{l}_{\alpha}, \mathfrak{h}_{\alpha}, \mathfrak{v}\right)\right)=B\left(\mathfrak{l}_{\alpha, \beta}, \mathfrak{h}_{\alpha, \beta}, \mathfrak{v}_{\alpha}\right) .
$$

Поэтому коммутативность алгебры $B\left(\mathfrak{l}_{\alpha}, \mathfrak{h}_{\alpha}, \mathfrak{v}\right)$ следует из коммутативности алгебры $B\left(\mathfrak{l}_{\alpha, \beta}, \mathfrak{h}_{\alpha, \beta}, \mathfrak{v}_{\alpha}\right)$ для любой точки $\beta \in(\operatorname{Ker} \hat{\alpha})^{*}$ общего положения.

\section{ЛиТЕРАТУРА}

1. Винберг Э. Б. Коммутативные однородные пространства и коизотропные симплектические действия. УМН, 56, вып. 1, 3-62 (2001).

2. Диксмье Ж. Универсальные обертывающие алгебры. Мир, М., 1978.

3. Микитюк И. В. Об интегрируемости инвариантных гамильтоновых систем с однородными конфигурационными пространствами. Матем. сб., 129, 514-534 (1986).

4. Duflo $M$. Open problems in representation theory of Lie groups. In: Conference on Analysis on homogeneous spaces (T. Oshima, ed.), August 25-30, Kataka, Japan, 1986, pp. 1-5.

5. Guillemin V., Sternberg S. Multiplicity-free spaces. J. Diff. Geom., 19, 31-56 (1984).

6. Knop F. Harish-Chandra homomorphism for reductive group actions. Ann. of Math. (2), Ser. 140, No. 2, 253-288 (1994).

Московский государственный университет, механико-математический факультет

Поступило в редакцию

e-mail: leo@rybnikov57.mccme.ru

29 мая 2002 г. 